\title{
Optimization of electrode position and electric pulse amplitude in electrochemotherapy
}

\author{
Anže Županič, Selma Čorović and Damijan Miklavčič \\ University of Ljubljana, Faculty of Electrical Engineering, Ljubljana, Slovenia
}

\begin{abstract}
Background. In addition to the chemotherapeutic drug being present within the tumor during electric pulse delivery, successful electrochemotherapy requires the entire tumor volume to be subjected to a sufficiently high electric field, while the electric field in the surrounding healthy tissue is as low as possible to prevent damage. Both can be achieved with appropriate positioning of the electrodes and appropriate amplitude of electric pulses.

Methods. We used 3D finite element numerical models and a genetic optimization algorithm to determine the optimum electrode configuration and optimum amplitude of electric pulses for treatment of three subcutaneous tumor models of different shapes and sizes and a realistic brain tumor model acquired from medical images.

Results. In all four tumor cases, parallel needle electrode arrays were a better choice than hexagonal needle electrode arrays, since their utilization required less electric current and caused less healthy tissue damage. In addition, regardless of tumor geometry or needle electrode configuration, the optimum depth of electrode insertion was in all cases deeper than the deepest part of the tumor.

Conclusions. Our optimization algorithm was able to determine the best electrode configuration in all four presented models and with further improvement it could be a useful tool in clinical electrochemotherapy treatment planning.
\end{abstract}

Key words: electrochemotherapy; electroporation; subcutaneous tumor; finite element method; numerical modeling; optimization

\section{Introduction}

Electrochemotherapy (ECT) is an effective local tumor therapy performed by the administration of chemotherapeutic drugs followed by the application of local high-voltage electric pulses., ${ }^{12}$ The electric

Received 19 May 2008

Accepted 29 May 2008

Correspondence to: Prof. Dr. Damijan Miklavčič, University of Ljubljana, Faculty of Electrical Engineering, Tržaška 25, SI-1000 Ljubljana, Slovenia, Phone: +386 14768 456, Fax: +386 14264 658, E-mail: damijan.miklavcic@fe.uni-lj.si pulses cause transient structural changes (electroporation) of tumor cell membranes and thus increase the entrance of the chemotherapeutic drugs. This potentiates the chemotherapeutic effect and lowers the required drug dose. ${ }^{3}$ Numerous studies have demonstrated ECT to be a very efficient treatment in various tumor types; in recent years, it has become a treatment of choice for cutaneous and subcutaneous tumor nodules of different histologies. ${ }^{4-9}$

Two conditions have to be met for ECT to be efficient: 1) a sufficient amount of chemotherapeutic drug has to be present in the 
a)

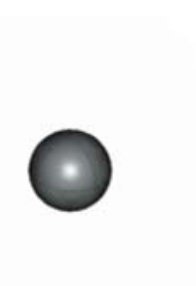

c)

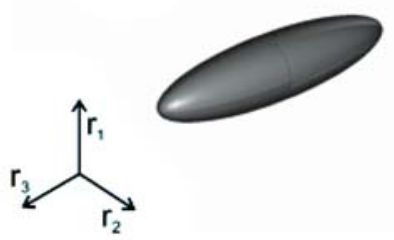

b)

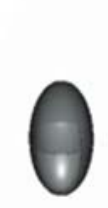

d)

Figure 1. 3D subcutaneous tumor geometries. a) sphere $\left.\left(\mathrm{r}_{1-3}=2 \mathrm{~mm}\right) ; \mathrm{b}\right)$ ellipsoid positioned deeper in tissue $\left(r_{1}=4 \mathrm{~mm}, \mathrm{r}_{2,3}=2 \mathrm{~mm}\right) ; \mathrm{c}$ ) ellipsoid $\left(\mathrm{r}_{1,2}=2 \mathrm{~mm}\right.$, $\left.r_{3}=8 \mathrm{~mm}\right) ; d$ ) realistic tumor geometry from medical images $\left(r_{1}=3.8 \mathrm{~mm}, r_{2}=2.4 \mathrm{~mm}, \mathrm{r}_{3}=2.6 \mathrm{~mm}\right)$.

target tissue, when the electric pulses are applied; 2) the electric pulses have to reversibly electroporate the entire tumor volume, which means that the electric field established by the pulses should be of a magnitude between the reversible and irreversible electroporation threshold $\left(\mathrm{E}_{\text {rev }}<\mathrm{E}<\mathrm{E}_{\text {irrev }}\right)$. The optimal ECT protocol should thus destroy all tumor cells, while minimising electrically induced damage to healthy tissue due to irreversible electroporation. This can be achieved by choosing the most suitable electrode configuration and the lowest amplitude of electric pulses that guarantees whole tumor electroporation. ${ }^{10,11}$ Finding the optimum treatment parameters is often difficult, since it requires a complete understanding of the treatment mechanisms. Since the electric field is one of the most important factors in ECT efficiency, modeling the electric field distribution is not only necessary for understanding the treatment, but is also a crucial step towards treatment planning. ${ }^{12-14}$ This study presents the first use of an ECT optimization algorithm on several different tumor geometries. a)

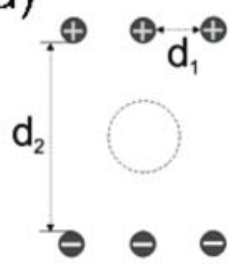

b)

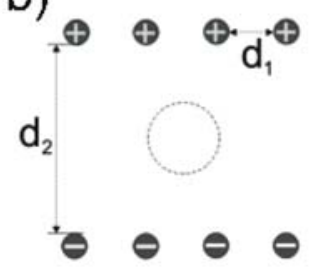

c)

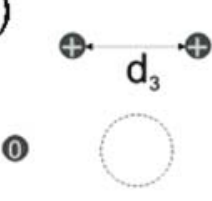

d)
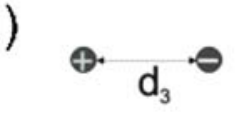

(0)

$\oplus$

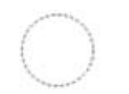

$\oplus$

Figure 2. Electrode geometries and polarities: a) three needle electrode pairs (3 pairs); b) four needle electrode pairs (4 pairs); c) hexagonal needle electrode array with two electrodes on positive potential, two on negative and two neutral $(2 \times 2)$; d) hexagonal needle electrode array with three electrodes on positive potential and three on negative potential $(3 \times 3)$. Distances between electrodes $d_{1-3}$ were among the optimized parameters in our optimization process. Diameter of all electrodes was $0.7 \mathrm{~mm}$.

The goal of our study was to optimize the electric field distribution in four different 3D subcutaneous tumor models (Figure 1) by optimizing the electrode configuration around the tumor tissue and the amplitude of the electric pulses for each of the four different electrode geometries that have been used in clinics in recent years (Figure 2). ${ }^{1,15}$ Optimization was performed using a combination of finite element numerical modeling and a genetic algorithm. All tumor/electrode cases were optimized for the following parameters: distances between electrodes (Figure 2), depth of electrode insertion and amplitude of electric pulses. Our optimization algorithm successfully found the best parameters in all cases and with some further improvement it could be a useful tool in clinical ECT treatment planning as well as in treatment planning of other electroporation based treatments. ${ }^{16-18}$ 


\section{Materials and methods}

Tissue properties and model geometry

Each model of a subcutaneous tumor consisted of two tissues: the target/tumor tissue and the surrounding healthy tissue. Four different tumor geometries were chosen, a small sphere, an ellipsoid positioned deeper in the tissue, an elongated ellipsoid and a realistic tumor geometry taken from a previous study and scaled for better comparison with the other tumor geometries (Figure 1). ${ }^{14}$ All tissues were considered isotropic and homogeneous, the assigned conductivity values being $0.4 \mathrm{~S} / \mathrm{m}$ for the tumors and $0.2 \mathrm{~S} / \mathrm{m}$ for the healthy tissue. These values describe the conductivity at the end of the electroporation process. ${ }^{19}$ The values were chosen in accordance with previous measurements of tumor and tissue conductivity and models of subcutaneous tumor and skin electroporation. ${ }^{13,16,20}$ The electric field distribution was calculated for three different electrode geometries: two different parallel needle electrode arrays (Figure 2a,b) and a hexagonal electrode array with two different electrode polarities (Figure 2c,d). These geometries and polarities were chosen because they are frequently used in ECT research and therapy.

\section{Numerical modeling}

Numerical calculations were performed with the commercial finite element software package COMSOL Multiphysics 3.4 (COMSOL AB, Sweden). The electric field distribution in the tissue, caused by the electroporative pulse, was determined by solving the Laplace equation for static electric currents:

$$
-\nabla \cdot(\sigma \cdot \nabla \varphi)=0
$$

where $\sigma$ and $\phi$ are the conductivity of the tissue and electric potential, respectively. The boundary conditions used in our cal-

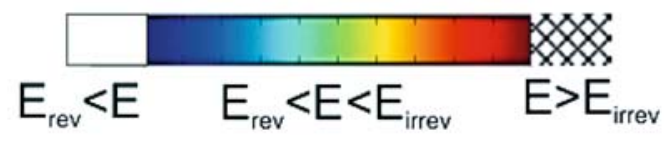

Figure 3. False color legend of Figs. 4, 5 indicating the degree of tissue permeabilization. The white region represents insufficiently permeabilized regions of tissue $\left(\mathrm{E}<\mathrm{E}_{\mathrm{rev}}\right)$ and the patterned region represents irreversibly permabilized regions of tissue $\left(\mathrm{E} \geq \mathrm{E}_{\text {irrev }}\right)$.

culations were a constant potential on the surface of the electrodes and electric insulation on all outer boundaries of the model.

The electric field distributions obtained in our models were displayed in the range from the reversible $\mathrm{E}_{\text {rev }}=400 \mathrm{~V} / \mathrm{cm}$ to the irreversible electroporation threshold value $\mathrm{E}_{\text {irrev }}=900 \mathrm{~V} / \mathrm{cm}$ (Figure 3). These values were taken from a previously published study, in which we estimated them by comparing in vivo measurements and numerical modeling of electroporation of a subcutaneous tumor. ${ }^{13,21}$

\section{Optimization}

The genetic algorithm ${ }^{22}$ was written in MATLAB 2007a (Mathworks, USA) and was run together with the finite element model using a link between MATLAB and COMSOL. The initial population of possible solutions was generated randomly, taking into account the following model constraints: range of distances between electrodes $\left(\mathrm{d}_{1}: 0.7-4.0 \mathrm{~mm} ; \mathrm{d}_{2}: 3.4-5.0 \mathrm{~mm}\right.$; $\left.\mathrm{d}_{3}: 1.3-5.0 \mathrm{~mm}\right)$, range of depths of electrode insertion into tissue $(-1.0-5.0 \mathrm{~mm}$ below the tumor) and range of amplitudes of electric pulses (1-1200 V). These constraints were chosen so that the calculation domain size, COMSOL meshing capabilities and oncology experts' demands for a safety margin ${ }^{23}$ when treating solid tumors, were all respected. Solutions for reproduction were selected proportionally to their fitness, according to the fitness function: 
Table 1. Optimized distances between electrodes $\left(\mathrm{d}_{1-3}\right)$, depth of electrode insertion below the tumor and amplitude of electric pulse (U) are given for all analyzed tumor models and electrode geometries. Qualities of individual optimized solutions are described by the calculated values of total electric current through tissue (I), fraction of reversibly permeabilised target tissue $\left(\mathrm{V}_{\text {Trev }} / \mathrm{V}_{\mathrm{T}}\right)$ and normalized volume of damaged healthy tissue $\left(\mathrm{V}_{\text {Hirrev }} / \mathrm{V}_{\text {sph }}\right)$.

\begin{tabular}{ccccccccc}
\hline Tumor $\begin{array}{c}\text { Electrode } \\
\text { geometry }\end{array}$ & $\mathbf{d}_{1}[\mathbf{m m}]$ & $\mathbf{d}_{2}[\mathbf{m m}]$ & $\mathbf{d}_{3}[\mathbf{m m}]$ & $\begin{array}{c}\text { Insertion } \\
\text { depth }[\mathbf{m m}]\end{array}$ & $\mathbf{U}[\mathbf{V}]$ & $\mathbf{I}[\mathbf{A}]$ & $\mathbf{V}_{\text {Trev }} / \mathbf{V}_{\mathrm{T}} \mathbf{V}_{\text {Hirrev }} / \mathbf{V}_{\text {sph }}$ \\
\hline 3 pairs & 0.70 & 3.4 & & 1.1 & 210 & 0.45 & 1 & 1.00 \\
4 pairs & 0.70 & 3.4 & & 0.9 & 210 & 0.52 & 1 & 1.03 \\
$3 \times 3$ & & & 1.3 & 0.3 & 200 & 0.55 & 1 & 3.58 \\
$2 \times 2 \times 2$ & & & 1.3 & 0.3 & 220 & 0.32 & 1 & 1.77 \\
\hline 3 pairs & 0.70 & 3.4 & & 0.9 & 220 & 0.65 & 1 & 1.59 \\
4 pairs & 0.70 & 3.6 & & 0.9 & 220 & 0.75 & 1 & 1.39 \\
$3 \times 3$ & & & 1.3 & 0.3 & 210 & 0.89 & 1 & 6.31 \\
$2 \times 2 \times 2$ & & & 1.3 & 0.7 & 220 & 0.47 & 1 & 2.51 \\
\hline 3 pairs & 2.60 & 3.4 & & 0.9 & 320 & 0.88 & 1 & 7.40 \\
4 pairs & 1.60 & 3.4 & & 0.7 & 320 & 0.96 & 1 & 7.08 \\
$3 \times 3$ & & & 4.3 & 0.5 & 550 & 1.19 & 1 & 15.84 \\
$2 \times 2 \times 2$ & & & 4.6 & 0.1 & 1160 & 1.25 & 1 & 31.22 \\
\hline 3 pairs & 0.75 & 3.4 & & 0.9 & 270 & 0.65 & 1 & 3.17 \\
4 pairs & 0.70 & 3.4 & & 0.7 & 270 & 0.70 & 1 & 3.39 \\
$3 \times 3$ & & & 1.8 & 1.1 & 320 & 1.07 & 1 & 11.44 \\
$2 \times 2 \times 2$ & & & 1.6 & 0.9 & 320 & 0.55 & 1 & 5.45 \\
\hline
\end{tabular}

$F=12+100 \cdot V_{\text {Trev }}-10 \cdot V_{\text {Hirrev }}-V_{\text {Hrev }}-V_{\text {Tirrev , }}$

where $\mathrm{F}$ stands for fitness, $\mathrm{V}_{\text {Trev }}$ and $\mathrm{V}_{\text {Tirrev }}$ stand for the tumor volume subjected to the local electric field above $E_{\text {rev }}$ and above $E_{\text {irrev, }}$ and $V_{\text {Hrev }}$ and $V_{\text {Hirrev }}$ stand for the volume of healthy tissue subjected to the local electric field above $\mathrm{E}_{\mathrm{rev}}$ and above $\mathrm{E}_{\text {irrev, }}$ respectively. The weights in the fitness function were set according to the importance of the individual parameters for efficient ECT. Namely, $V_{\text {Trev }}$ is crucial for efficient ECT, so its weight is largest (100) in comparison to the weight of $\mathrm{V}_{\text {Hirrev }}(10)$, which was in turn larger than the weights of $\mathrm{V}_{\mathrm{Hrev}}$ and $\mathrm{V}_{\text {Tirrev }}$ since their significance for successful electrochemotherapy is still debated. Other weight values that kept a similar ratio gave similar results. The integer 12 is present only to ensure that the fitness function is always positive.

The selected solutions reproduced by cross-over or by mutation. The genetic algorithm was terminated after 100 generations, when the fitness of the highest ranking solution usually reached a plateau. The average computation time of the algorithm was two hours on a standard desktop PC (Windows XP, 3.0 GHz, 1 GB RAM).

\section{Results}

The optimized parameters of electrochemotherapy (ECT) for all tumor/electrode cases are given in Table 1 . The optimum distance 
a)

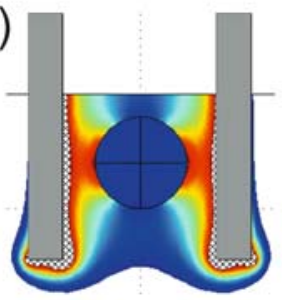

b)

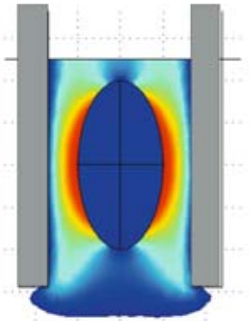

c)

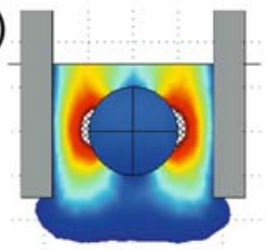

d)

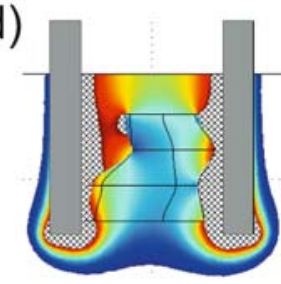

Z

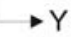

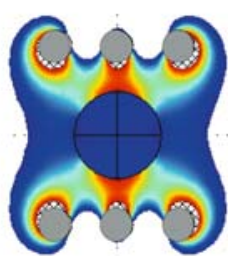
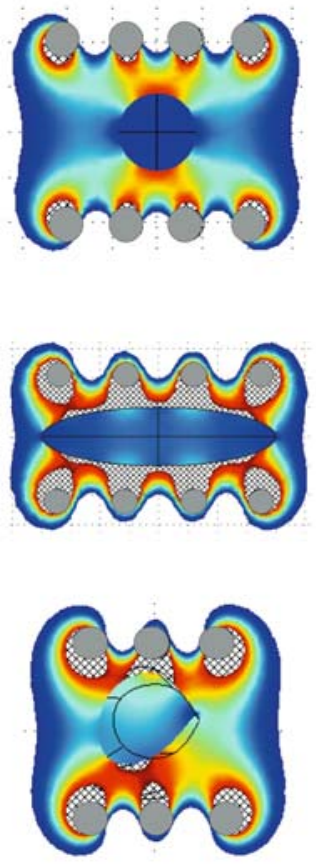

Y

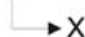

Figure 4. Electric field distribution for the optimized models of subcutaneous tumors is shown. In each case, only the best electrode configuration is given: a) three needle pairs for the spherical tumor; b) four needle pairs for the ellipsoid; c) four needle pairs for the ellipsoid deeper in tissue; d) three needle pairs for the realistic tumor. The electric distribution is shown in two central perpendicular planes: $\mathrm{YZ}$ and $\mathrm{XY}$ both passing through the center of the tumor. Corresponding values of parameters are given in Table 1.

between electrodes in a parallel row $\left(\mathrm{d}_{1}\right)$ was similar for all tumor models, except, due to its size, for the elongated ellipsoid tumor geometry, for which successful electroporation required the electrodes to be

further apart. The electrodes were as close to each other as possible considering the parameter constraints, which guaranteed that the electric field distribution in the target tissue was homogeneous as possible (comparison of Figure $4 \mathrm{~b}$ and Figure $4 \mathrm{c}$ ). The optimum distance between electrode rows $\left(d_{2}\right)$ was also similar for all tumor geometries and as small as possible, the reason being that small inter-electrode distances required a lower voltage to ensure electroporation, thus also requiring less electric energy and causing less damage to tissue. The same is true for the distance between electrodes in a hexagonal array $\left(\mathrm{d}_{3}\right)$, the reason this time being a combination of both homogeneity of the local electric field and lower required voltage. In contrast, the optimum depth of electrode insertion varied with the tumor and electrode geometry. Nevertheless, the optimum position for the electrodes was in all cases below the tumor. The optimum electric pulse amplitude did not differ much in cases of a spherical tumor and ellipsoid tumor deep in tissue but in other tumor geometries, parallel electrode arrays required considerably lower amplitudes than their hexagonal counterparts.

We compared the quality of the optimized solution in terms of total electric current through the tissue and extent of healthy tissue damage (Table $1-\mathrm{V}_{\text {Hirrev }} /$ $\mathrm{V}_{\text {sph }}$ ). We normalized the volumes of irreversibly electroporated tumor with the volume of a spherical tumor better to compare the amount of tissue damage between individual treatment cases. Parallel electrode arrays gave better results for all four tumor geometries. Three needle pairs always resulted in less total electric current. However, four needle pairs produced a more homogeneous field, which, in combination, caused three needle pairs to be a slightly better choice (less healthy tissue damage) for the spherical and the realistic 
a)

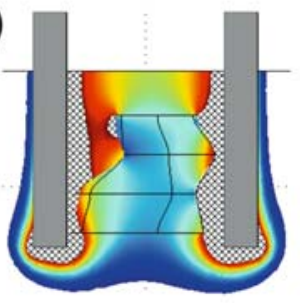

b)

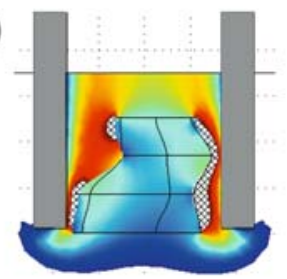

c)

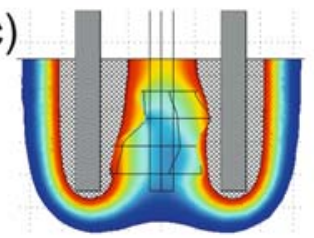

d)
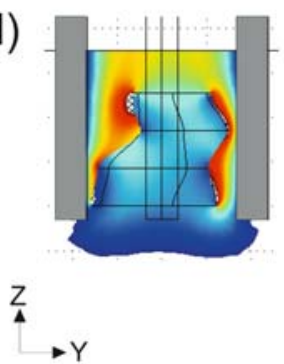

Figure 5. Electric field distribution for the optimized model of the realistic tumor with a) three needle pairs; b) four needle pairs; c) $3 \times 3$ hexagonal needle electrode array; d) $2 \times 2 \times 2$ hexagonal needle electrode array is shown. The electric distribution is shown in two central perpendicular planes: $\mathrm{YZ}$ and $\mathrm{XY}$ both passing through the center of the tumor. Corresponding values of parameters are given in Table 1.

tumor geometry and four needle pairs to be slightly better for the other two geometries. The best electrode configurations for all tumor geometries and the corresponding electric field distributions are shown in Figure 4. Hexagonal electrodes caused considerably more healthy tissue damage $\left(\mathrm{E}>\mathrm{E}_{\text {irrev }}\right)$ than parallel electrodes, which can be seen in Figure 5 for the realistic tumor geometry. The $3 \times 3$ hexagonal electrode array caused more healthy tissue damage than the other three geometries and also required the highest total electric current, mostly because the electric current ran between the closest positive and negative electrodes, instead of through the target tissue (Figure 5).

\section{Discussion}

The aim of our study was to optimize the electrode configuration around the target tissue and electric pulse amplitude for ECT of four 3D models of subcutaneous tumors treated with four different needle electrode array geometries. In all 16 cases, the optimization resulted in reversible electroporation of the entire tumor (Table $1: \mathrm{V}_{\text {Trev }} / \mathrm{V}_{\mathrm{T}}=1$ ), which was the parameter with the highest weight in our fitness function. At the same time, the damage to healthy tissue was minimal. When treating a spherical tumor, only a volume of healthy tissue equal to the tumor volume was irreversibly electroporated (Table 1: $\mathrm{V}_{\text {Hirrev }} / \mathrm{V}_{\text {sph }}$ ). Treatment of larger tumors caused more healthy tissue damage.

The usefulness of numerical modeling in predicting electroporation outcomes has already been demonstrated. ${ }^{14,15,19,24-26}$ We examined the adequacy for ECT of needle electrode array geometries by calculating the values of total electric current through the model (must be as low as possible to avoid nerve stimulation ${ }^{27}$ and not exceed the capacities of the electric pulse generator ${ }^{28}$ ) and volumes of reversibly and irreversibly electroporated tumor tissue and healthy tissue. Three-needle electrode pairs were best for the spherical and the realistic tumor geometry; they required the lowest total electric current and caused only a small volume of healthy tissue to be irreversibly electroporated (healthy tissue damage) (Figure 4). 
Four-needle electrode pairs caused the least healthy tissue damage in the other tumor geometries, but they required more electric current (Figure 4), confirming previous results of our group - more electrodes mean a more invasive procedure, higher required current and lower required voltage to obtain the same target tissue coverage. Parallel electrode arrays gave much better results than the $2 \times 2$ and $3 \times 3$ hexagonal needle electrode arrays, mostly because they induced a much more homogeneous field and, consequently, a lower electric current density.

Our work built on a previous study by our group that optimized the distance and voltage between electrodes for a realistic brain tumor (the same tumor geometry that we used in a scaled form in this study). ${ }^{14}$ Our present study took optimization one step further by optimizing for four different electrode geometries and for two additional parameters, i.e. distance between electrodes in a row and depth of electrode insertion, which lead to perhaps the most important practical result. It is very difficult to guess the best possible insertion depth, since it depends in complex ways on tumor geometry, electrode geometry, electroporation thresholds and the conductivities of tumor and healthy tissue. However, based on our results, electrodes should always be inserted deeper than the deepest part of the tumor (Table 1).

We chose a genetic algorithm as the optimization method, since different linear and non-linear constraints, such as the technical limitations of the high-voltage electric pulse generator (maximum output voltage and current) can be easily taken into account. A genetic algorithm also allows optimization of a large number of continuous, discrete and categorical parameters, e.g. type of electrodes and can give as a result many solutions of similar quality, which can nevertheless be topologically very different. This gives the treating physician more alterna- tives for the positioning of electrodes, which can be very valuable if some of them are not easy to access. The major drawback of a genetic algorithm is the relatively long computation time. However, since it can be considerably shortened by using a more powerful computer or by making the optimization parameters discrete instead of continuous, we do not consider this to be a significant issue and believe that this approach is well suited to the problem being addressed.

Even though our algorithm gives good results, several challenges remain to be addressed before it can be used for treatment planning of ECT. We must determine the most appropriate level of complexity of our numerical models. In this study, we did not take into account changes to tissue conductivity due to electroporation, the possibility of several consecutive pulses being used, of changing the electric field orientation or of moving the electrodes during treatment of a larger tumor; all of which options must be considered in the future. ${ }^{13,15,25}$ Another crucial development would be an algorithm that would convert medical images of the treatment area into 3D structures ready to import into numerical modeling software.

In conclusion, we demonstrated that numerical modeling and optimization can be efficiently combined to control the extent of tissue electroporation in ECT and to produce the optimum electrode configuration and amplitude of electric pulses. Our algorithm is a step towards effective treatment planning, not only in clinical ECT, but also in other electroporation based treatments, such as gene electrotransfer, transdermal drug delivery and irreversible tumor ablation.16-18

\section{Acknowledgements}

This research was supported by the Slovenian Research Agency. 


\section{References}

1. Marty M, Sersa G, Garbay JR, et al. Electrochemotherapy - An easy, highly effective and safe treatment of cutaneous and subcutaneous metastases: Results of ESOPE (European Standard Operating Procedures of Electrochemotherapy) study. Eur J Cancer Suppl Nov 2006; 4: 3-13.

2. Sersa G. The state-of-the-art of electrochemotherapy before the ESOPE study; advantages and clinical uses. Eur J Cancer Suppl 2006; 4: 52-9.

3. Domenge C, Orlowski S, Luboinski B, DeBaere T, Schwaab G, Belehradek J, et al. Antitumor electrochemotherapy - New advances in the clinical protocol. Cancer 1996; 77: 956-63.

4. Heller R, Gilbert R, Jaroszeski MJ. Clinical applications of electrochemotherapy. Adv Drug Deliv Rev 1999; 35: 119-29.

5. Gothelf A, Mir LM, Gehl J. Electrochemotherapy: results of cancer treatment using enhanced delivery of bleomycin by electroporation. Cancer Treat Rev 2003; 29: 371-87.

6. Tijink BM, De Bree R, Van D, Leemans CR. How we do it: Chemo-electroporation in the head and neck for otherwise untreatable patients. Clin Otolaryngol 2006; 31: 447-51.

7. Mir LM, Gehl J, Sersa G, et al. Standard operating procedures of the electrochemotherapy: Instructions for the use of bleomycin or cisplatin administered either systemically or locally and electric pulses delivered by the Cliniporator (TM) by means of invasive or non-invasive electrodes. Eur J Cancer Suppl 2006; 4: 14-25.

8. Snoj M, Rudolf Z, Cemazar M, Jancar B, Sersa G. Successful sphincter-saving treatment of anorectal malignant melanoma with electrochemotherapy, local excision and adjuvant brachytherapy. AntiCancer Drugs 2005; 16: 345-8.

9. Sersa G, Cemazar M, Miklavcic D, Rudolf Z. Electrochemotherapy of tumours. Radiol Oncol 2006; 40: 163-74.

10. Miklavcic D, Beravs K, Semrov D, Cemazar M, Demsar F, Sersa G. The importance of electric field distribution for effective in vivo electroporation of tissues. Biophys J 1998; 74: 2152-8.

11. Miklavcic D, Semrov D, Mekid H, Mir LM. A validated model of in vivo electric field distribution in tissues for electrochemotherapy and for DNA electrotransfer for gene therapy. Biochim Biophys Acta 2000; 1523: 73-83.
12. Miklavcic D, Corovic S, Pucihar G, Pavselj N. Importance of tumour coverage by sufficiently high local electric field for effective electrochemotherapy. Eur J Cancer Suppl 2006; 4: 45-51.

13. Pavselj N, Bregar Z, Cukjati D, Batiuskaite D, Mir LM, Miklavcic D. The course of tissue permeabilization studied on a mathematical model of a subcutaneous tumor in small animals. IEEE Trans Biomed Eng 2005; 52: 1373-81.

14. Sel D, Lebar AM, Miklavcic D. Feasibility of employing model-based optimization of pulse amplitude and electrode distance for effective tumor electropermeabilization. IEEE Trans Biomed Eng 2007; 54: 773-81.

15. Gilbert RA, Jaroszeski MJ, Heller R. Novel electrode designs for electrochemotherapy. Biochim Biophys Acta 1997; 1334: 9-14.

16. Cukiati D, Batiuskaite D, Andre F, Miklavcic D, Mir LM. Real time electroporation control for accurate and safe in vivo non-viral gene therapy. Bioelectrochemistry 2007; 70: 501-7.

17. Pliquett UF, Vanbever R, Preat V, Weaver JC. Local transport regions (LTRs) in human stratum corneum due to long and short 'high voltage' pulses. Bioelectrochem Bioener 1998; 47: 151-61.

18. Al Sakere B, Bernat C, Connault E, Opolon O, Rubinsky B, Davalos R, et al. Tumour ablation with irreversible electroporation. PLOS ONE 2007:11.

19. Corovic S, Pavlin M, Miklavcic D. Analytical and numerical quantification and comparison of the local electric field in the tissue for different electrode configurations. Biomed Eng Online 2007; 6.

20. Miklavcic D, Pavselj N, Hart FX. Electric properties of tissues. Wiley Encyclopedia of Biomedical Engineering. New York: John Wiley \& Sons; 2006.

21. Semrov D, Miklavcic D. Calculation of the electrical parameters in electrochemotherapy of solid tumours in mice. Comput Biol Med 1998; 28: 439-48.

22. Holland JH. Adaptation in Natural and Artificial Systems: An Introductory Analysis with Applications to Biology, Control, and Artificial Intelligence. Cambridge: MIT Press; 1992.

23. Gehl J, Geertsen PF. Palliation of haemorrhaging and ulcerated cutaneous tumours using electrochemotherapy. Eur J Cancer Suppl 2006; 4: 35-7.

24. Gehl J, Sorensen TH, Nielsen K, Raskmark P, Nielsen SL, Skovsgaard T, et al. In vivo electroporation of skeletal muscle: threshold, efficacy and relation to electric field distribution. Biochim Biophys Acta 1999; 1428: 233-40. 
25. Sersa G, Cemazar M, Semrov D, Miklavcic D. Changing electrode orientation improves the efficacy of electrochemotherapy of solid tumors in mice. Bioelectrochem Bioener 1996; 39: 61-6.

26. Sel D, Mazeres S, Teissie J, Miklavcic D. Finite-element modeling of needle electrodes in tissue from the perspective of frequent model computation. IEEE Trans Biomed Eng 2003; 50: 1221-32.

27. Zupanic A, Ribaric S, Miklavcic D. Increasing the repetition frequency of electric pulse delivery reduces unpleasant sensations that occur in electrochemotherapy. Neoplasma 2007; 54: 246-50.

28. Puc M, Corovic S, Flisar K, Petkovsek M, Nastran $\mathrm{J}$, Miklavcic D. Techniques of signal generation required for electropermeabilization. Survey of electropermeabilization devices. Bioelectrochemistry 2004; 64: 113-24. 